\title{
Susceptibility of Streptococcus pneumoniae causing bacterial meningitis in children in Yaoundé (Cameroon): results of a surveillance site
}

\begin{abstract}
Background: Streptococcus pneumoniae (S. pneumoniae) is the main cause of febrile conditions especially among children below two years and the elderly above 65 years old worldwide. In Cameroon, the frequency of $S$. pneumoniae as the cause of meningitis varied from $57.2 \%$ to $56.2 \%$ from 2001 to 2012 . The aim of this study was to determine the susceptibility to antibiotics of $S$. pneumoniae involved in meningitis.

Methods: A cross-sectional study was carried out during a four-year period from April 2012 to March 2016. Cerebrospinal fluid (CSF) was collected by clinicians from children less than 5 years with clinically suspected bacterial meningitis at the Mother and Child Center, Chantal Biya Foundation (MCC-CBF), in Yaounde. A probable case is a suspected clinical case with the CSF showing at least one of the following elements: turbid appearance; leukocytes $\geq 10 / \mathrm{mm}^{3}$, elevated protein $(>100 \mathrm{mg} / \mathrm{dl})$ or decreased glucose $(<40 \mathrm{mg} / \mathrm{dl})$. Cases are confirmed when isolates are identified. The identification of $S$. pneumoniae was done by soluble antigens, the Binax Now ${ }^{\circledR}$ test, Gram stain, catalase test, optochin test, bile solubility and molecular serotyping. The antimicrobial susceptibility testing was carried out using the disc diffusion method on Mueller-Hinton medium with $10 \%$ sheep blood and the minimal inhibitory concentration was found using the Etest ${ }^{\circledR}$.
\end{abstract}

Results: Out of 6449 clinically suspected cases of bacterial meningitis, 384 were probable and 91 cases were confirmed. Most were isolated in children less than one year old: 54/91 (59.34\%) and in boys 51/91 (56.04\%). These incidences occurred mainly during the dry season. In the confirmed cases, $60(65.93 \%)$ were due to $S$. pneumoniae, $9(9.89 \%)$ due to Haemophilus influenzae B, $2(2.19 \%)$ due to Neisseria meningitis and $20(21.97 \%)$ due to other germs. Among the serotyped pneumococci 10/51 (19.60\%) belonged to serotype $6 \mathrm{~A} / 6 \mathrm{~B}$. Of 35 viable isolates of $S$. pneumoniae, 15 (42.85\%) were resistant to penicillin G and $3(8.57 \%)$ to ceftriaxone.

Conclusion: S. pneumoniae is the main etiology of childhood meningitis in Yaounde. The frequency of resistance to penicillin $\mathrm{G}$ was high, while moderate to ceftriaxone.

Keywords: Streptococcus pneumoniae, bacterial meningitis, serotype 6A/6B, susceptibility, Cameroon
Volume 7 Issue 5 - 2019

\author{
Josiane Myriam Meli Tiabou,' Angeline \\ Boula, ${ }^{2}$ Jean Taguebue, ${ }^{2}$ Hortense Gonsu \\ Kamga,' John Njuma Libwea, ${ }^{3}$ Sandrine \\ Bebey, ${ }^{2}$ Madeleine Ngo Baleba, ${ }^{2}$ Eric Nkolo, ${ }^{2}$ \\ Emilia Lyonga-Mbamyah,' David Mekontso, ${ }^{4}$ \\ Marie Kobela, ${ }^{5}$ Arianne Nzouankeu, ${ }^{6}$ Regis \\ Tanga Tanga, ${ }^{7}$ Brenda Kwambana Adams, ${ }^{8}$ \\ Jason M. Mwenda, ${ }^{9}$ Martin Antonio, ${ }^{8}$ Paul \\ Koki Ndombo, ${ }^{1,2}$ Sinata Koulla Shiro,' Wilfred \\ Mbacham $^{10}$ \\ 'Department of Microbiology and Infectious Diseases, Faculty \\ of Medicine and Biomedical Sciences, University of Yaoundé I, \\ Cameroon \\ 2Mother and Child Center, Chantal Biya Foundation, Cameroon \\ ${ }^{3}$ University of Tampere, Finland \\ ${ }^{4} \mathrm{WHO}$, Cameroon, \\ ${ }^{5}$ Enlarged Program of Immunization, Cameroon \\ ${ }^{6}$ Centre Pasteur, Cameroon, \\ 7 Department of Pharmacy, Faculty of Medicine and \\ Pharmaceutical Sciences, University of Douala, Cameroon \\ ${ }^{8}$ Medical Research Council Unit, Gambia \\ 'WHO Regional Office for Africa, Republic of Congo \\ ${ }^{10}$ Department of Physiological Sciences/Biochemistry, Faculty \\ of Medicine and Biomedical Sciences, University of Yaounde I, \\ Cameroon
}

Correspondence: Wilfred F Mbacham, Professor of Public Health Biotechnology, University of Yaounde I, Cameroon, Box 8094, Yaounde, Cameroon, Tel +237-677579180,

Emailwfmbacham@yahoo.com

Received: August 19,2019 | Published:September 13,2019

\begin{abstract}
Abbreviations: S, Streptococcus; WHO, world health organization; N. m, Neisseria meningitis; Hi, Haemophilus. influenzae; CSF, cerebrospinal fluid; PCV, pneumococcal conjugate vaccine; EPI, Expanded Immunization Program; MCC-CBF, Mother and Child Center, Chantal Biya Foundation; PBM, pediatric bacterial meningitis; CDC, centers for disease control and prevention; TI, trans-isolate; TSA, trypticase soy agar; $\mathrm{BHI}$, brain heart infusion; RRL, regional reference laboratory ; MRCG, medical research council, Gambia; CLSI, clinical and laboratory standards institute; MIC, minimal inhibitory concentration; ATCC, American type culture collection; PCR, polymerase chain reaction; PNSSP, penicillin-non susceptible S. pneumoniae; E. coli, Escherichia coli; IPD, invasive pneumococcal diseases; DLMEP, direction de la Lutte contre la Maladie, les Épidémies et les Pandémies (Cameroon's Department of Disease Control); NICD, national institute for communicable diseases; CRDM, centre for respiratory diseases and meningitis; BTC, biotechnology centre; UYI, University of Yaounde I
\end{abstract}

\section{Introduction}

S. pneumoniae is a commensal bacterium of the human upper respiratory tract. However, it can cause invasive diseases such as septicemia, pneumonia, meningitis, and non-invasive diseases like otitis. ${ }^{1}$ The World Health Organization (WHO) estimated that about 800,000 under-five deaths occurring yearly were due to pneumococcal infections. Ninety percent of these deaths were reported to be registered in developing countries, with pneumococcal meningitis presenting a high case fatality rate $(36 \%-66 \%)$ in the epidemic meningitis belt. ${ }^{2}$ Furthermore, this strain was found to be more difficult to treat than meningococcal meningitis and was frequently associated with severe sequelae. ${ }^{3}$ Although to date, largescale epidemics of pneumococcal meningitis have not been observed in sub-Saharan Africa, its responsibility in the seasonal fluctuation of meningitis incidence in the meningitis belt area follows closely that due to Neisseria meningitis (N. m), known as the meningococcus. Incidence in the dry season has been up to ten times higher than in the 
wet season. ${ }^{3}$ From 2004 to 2013, S. pneumoniae was detected in over 4000 cases of bacterial meningitis in the meningitis belt, representing $27 \%$ of confirmed cases. ${ }^{4}$ Cerebrospinal fluid (CSF) was collected from only a few suspected cases, and because laboratory diagnosis is often difficult, the true number of cases was likely to be much higher.

Koulla, ${ }^{5}$ in 1997 , found S. pneumoniae in $58.3 \%$ of known etiologies of bacterial meningitis in the Yaounde Central Hospital. Further studies conducted in Cameroon from 2001 to 2014 reported an average variation of $S$. pneumoniae frequency of over $48.25 \%{ }^{6-8}$ It was within this framework that the 13-valent pneumococcal conjugate vaccine (PCV13, Prevenar13 ${ }^{\mathrm{R}}$, Pfizer Inc) was introduced into the Expanded Immunization Program (EPI) in 2011. ${ }^{9}$ Kwambana found that Pneumococci expressing the serotype 1 capsule dominated the West African pneumococcal meningitis outbreaks. ${ }^{10}$ Some studies reported that strains isolated from the upper respiratory tract of subjects in the Center Region of Cameroon were relatively sensitive to $\beta$-lactamines. ${ }^{11}$ The increasing resistance of $S$. pneumoniae to penicillin and other beta-lactamines raises enormous challenges in treatment regimens. ${ }^{12}$ Thus, the dissemination of $S$. pneumoniae strains with decreased sensitivity to penicillin constitutes a serious public health concern. ${ }^{13}$ This calls for the establishment of epidemiological surveillance at every stratum of the health sector to enhance disease control and prevention. ${ }^{14}$ The aim of the present project was to study the antibiotic resistance of some $S$. pneumoniae strains isolated from meningitis patients in the Mother and Child Center, Chantal Biya Foundation (MCC-CBF) Yaounde.

\section{Materials and methods}

\section{Study design and data source}

This was a cross-sectional survey that covered a period of 4 years (from April 2012 to March 2016). Register-based data from the Pediatric Bacterial Meningitis (PBM) Surveillance Network at the MCC-CBF Yaounde was used. This network was established in 2002 and followed up by WHO Regional Office for Africa (WHOAFRO) and the Centers for Disease Control and Prevention (CDC). It involved children less than five years old admitted in hospital for suspected meningitis. The samples and isolates were cultured for identification and antibiotic susceptibility.

Ethical clearance was obtained from the Cameroon National Ethics Committee for Research on Human Health (Ref N²016/04/749/ $\mathrm{CE} / \mathrm{CNERSH} / \mathrm{SP}$ ). Inform consent was sought from the parents or guardians of the surveillance participants.

\section{Case definition}

Suspected case: Any child aged 0-59 months admitted to MCC-CBF with sudden onset of fever $\left(>38.5^{\circ} \mathrm{C}\right.$ rectal or $38.0^{\circ} \mathrm{C}$ axillary) and one of the following signs: seizure, altered consciousness with no alternative diagnosis, or other meningeal sign; or every patient aged under 5 years hospitalized with a clinical diagnosis of meningitis. ${ }^{15}$

Probable case: A suspected meningitis case (as defined above) with cerebrospinal fluid (CSF) examination showing at least one of the following:

a. Turbid appearance (including cloudy, purulent);

b. Leukocytosis $\left(>10\right.$ cells $\left./ \mathrm{mm}^{3}\right)$ and either an elevated protein $(>$ $100 \mathrm{mg} / \mathrm{dl})$ or decreased glucose $(<40 \mathrm{mg} / \mathrm{dl})$ level..$^{15}$
Confirmed case: A suspected meningitis case that is laboratoryconfirmed by growing (culturing) or identifying a bacterial pathogen in the CSF (by Gram stain, antigen detection, and/or immunochromatography). ${ }^{15}$ It can be accompanied by a positive blood culture.

\section{Sample collection, bacterial identification, serotyping and interpretation}

CSF collected by clinicians from suspected meningitis participants were received and transferred immediately to the laboratory for bacteriological analysis. The latex agglutination technique (BIO-RAD Pastore $^{\text {tm }}$ Meningitis) was used to detect soluble antigens of $N . m, S$. pneumoniae and $H$. influenzae type b. The immunochromatographic test (Binax Now ${ }^{\circledR}$ Alere) for the rapid detection of $S$. pneumoniae antigens was also carried out on positive soluble antigens.

Bacterial culture was done in appropriate test media for confirmation and isolation of cases. A quantity of $1 \mathrm{ml}$ of CSF was inoculated into the Trans-Isolate (TI) medium (HiCombi ${ }^{\mathrm{TM}}$ Dual Performance Trans Isolate Medium LQ109) where a needle blocked with cotton was inserted for ventilation. The TI medium was well mixed, and a drop was transferred into Trypticase Soy Agar (TSA) $+10 \%$ of fresh sheep blood and chocolate agar plus Isovitalex, streaked and incubated at $37^{\circ} \mathrm{C}$ in a candle-extinction jar for 18 to 24 hours. The organisms were identified using conventional methods (colony morphology, Gram stain, optochin susceptibility, Binax Now ${ }^{\circledR}$, catalase test and bile solubility test for $S$. pneumoniae). ${ }^{16}$ Protein and glucose levels of the CSF were also measured. An aliquot of the specimens and strains conserved in Brain Heart Infusion (BHI) $+25 \%$ glycerol was shipped on dry ice $\left(-80^{\circ} \mathrm{C}\right)$ to the WHO Regional Reference Laboratory (RRL) hosted at the Medical Research Council, Gambia (MRCG) for serotyping. The rest of the CSF was aliquoted into cryotubes and conserved at $-80^{\circ} \mathrm{C}$ for later use. ${ }^{17}$

\section{Antibiotic susceptibility tests}

Disc diffusion susceptibility testing was carried out for 35 strains according to Clinical and Laboratory Standards Institute (CLSI) guidelines.. The antibiotics tested by this method were: oxacillin (Ox $1 \mu \mathrm{g})$, ampicillin (Ap $10 \mu \mathrm{g})$, cephalotin (KF30g), erythromycin (E, $15 \mathrm{UI})$, clindamycin (DA, $2 \mu \mathrm{g})$, vancomycin (VA, $30 \mu \mathrm{g})$, tetracycline

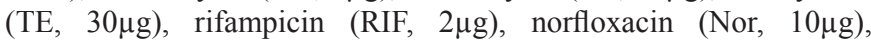
levofloxacin (LEVOF, $5 \mu \mathrm{g}$ ) and doxycycline (D, 30 $\mu \mathrm{g}$ ) (BLVD Disk Sensi-Disc BBL (Becton Dickinson)).

Ceftriaxone may be used to treat pneumococcal infections; however, reliable disk diffusion susceptibility tests with this antibiotic do not yet exist. Its in vitro activity is best determined using a Minimal Inhibitory Concentration (MIC) method. ${ }^{18}$ For the determination of the MIC of penicillin G, ceftriaxone and chloramphenicol for the same 35 strains, the gradient strip Etest ${ }^{\circledR}$ susceptibility method was used according to the manufacturer's instructions (BioMérieux, Marcy l'Étoile, France). ${ }^{19}$ The criteria for interpretation were those provided by the CLSI. ${ }^{20}$

\section{Quality control}

The reference strain, S. pneumoniae ATCC 49619, was included on each day of testing. Results of susceptibility testing were accepted if the results of the control strains were within published limits. 


\section{Statistical analysis}

The data obtained were entered into a data form and analyzed using Excel 2013 (Microsoft Office, USA) and SPSS16.0 (SPSS, Inc, Chicago, IL, USA). Two-way ANOVA (Tukey) and One-way ANOVA were used when appropriate.

\section{Results}

A total of 6449 CSF was received from subjects with clinically suspected meningitis from April 2012 to March 2016 within the context of surveillance of pediatric bacterial meningitis. Out of these, $384 / 6449(5,95 \%)$ were detected as being probable cases and only $91 / 384(23,69 \%)$ cases were confirmed. Of the 384 probable cases, $362(94.27 \%)$ were tested for soluble antigens and $45(12.43 \%)$ specimens were positive for $S$. pneumoniae.

The following 91 bacterial strains were identified: 60 (65.93\%) $S$. pneumoniae, 9 (9.89\%) H. influenzae type b, 2 (2.19\%) N. meningitis and 20 (21.97\%) other germs (10 Streptococcus type B, 3 Klebsiella pneumoniae, 2 E. coli, 2 Staphylococcus aureus, 1 Acinetobacter baumannii, 1 Serratia liquefasciens, and 1 Salmonella sp). Among the 60 identified $S$. pneumoniae, 45 were positive for soluble antigens and 51 were culture-positive strains.

Table 1 shows the demographic characteristics of children aged less than 5 years infected with bacterial meningitis. Of the 91 patients with positive CSF, 51 (56.04\%) were male and 40 (43.95\%) female. A twoway ANOVA was conducted and there was a statistically significant difference between the two groups, $F(4,4)=1389,519, P=.000$. The patients's median age was 14 months. Of these, 20 (26.50\%) were less than 6 weeks of age and 54 (59.34\%) were less than one year old. The age has a significant influence on the infection to the different bacteria $(\mathrm{P}<0,05)$. There were 15 deaths due to $S$. pneumoniae, giving a mortality rate of $16.48 \%$ The number of children who recovered after the bacterial infection is significantly higher than those who die $(\mathrm{P}<0,05)$. This mortality was higher in the first year of life $(10 / 15-66.66 \%)$ compared to the next four years $(5 / 15-33,34 \%)$. $S$. pneumoniae was shown to have a higher mortality rate than the other germs. Diagnosed meningitis cases showed clear seasonal variations, with three peaks in March, July and December, all within dry seasons. It was found that $19.60 \%(10 / 51)$ of the confirmed and serotyped pneumococcal meningitis cases were attributed to serotype $6 \mathrm{~A} / 6 \mathrm{~B}$ (Table2).

The global susceptibility profile (Tables $3 \& 4$ ) shows important resistance to penicillin $\mathrm{G}(42.85 \%)$, oxacillin $(51.42 \%)$, tetracycline $(60 \%)$, trimethoprim-sulfamethoxazole $(85.71 \%)$ and moderate resistance to ceftriaxone $(8.57 \%)$. Overall, $15(42.85 \%)$ of the isolates were non-susceptible to penicillin $G$ (penicillin-non susceptible $S$. pneumoniae [PNSSP]). Our isolates were highly susceptible to ampicillin (88.58\%), third-generation cephalosporins $(91.43 \%)$, rifampicin $(79.16 \%)$ and vancomycin $(100 \%)$.

Table I Demographic characteristics of children with confirmed bacterial meningitis

\begin{tabular}{|c|c|c|c|c|c|c|c|c|c|c|c|}
\hline \multirow{2}{*}{ Bacteria } & \multirow{2}{*}{$\begin{array}{l}\text { Number } \\
\text { of } \\
\text { isolates } \\
\text { n (\%) }\end{array}$} & \multicolumn{2}{|c|}{ Gender n (\%) } & \multicolumn{5}{|c|}{ Age of diagnosis (months) $n(\%)$} & \multicolumn{3}{|c|}{ Outcome n (\%) } \\
\hline & & Male & Female & $0-1.5$ & $1.6-5$ & $06-11$ & $12-23$ & 24-59 & Recovery & Death & Unknown \\
\hline H. influenzae & $9(9.89)$ & $5(55.55)$ & $4(44.44)$ & & $4(44.44)$ & $2(22.22)$ & I (II.II) & $2(22.22)$ & $4(44.44)$ & I (II.II) & $4(44.44)$ \\
\hline N. meningitis & $2(2.19)$ & $2(100)$ & 0 & $\mathrm{I}(50)$ & & $\mathrm{I}(50)$ & & $2(100)$ & I (50) & & I (50) \\
\hline $\begin{array}{l}\text { Streptococcus } \\
\text { B }\end{array}$ & $10(10.98)$ & $5(50)$ & $5(50)$ & $9(90)$ & & & $\mathrm{I}(10)$ & & $3(30)$ & $4(40)$ & $3(30)$ \\
\hline $\begin{array}{l}\text { K. } \\
\text { pneumoniae }\end{array}$ & $3(3.29)$ & & $3(100)$ & & & I (33.33) & & $2(66.66)$ & & I (33.33) & $2(66.66)$ \\
\hline E. coli & $2(2.19)$ & I (50) & I (50) & I (50) & & & & I (50) & I (50) & & I (50) \\
\hline S. aureus & $2(2.19)$ & I (50) & I (50) & I (50) & I (50) & & & & I (50) & & I (50) \\
\hline A. baumanii & I (1.09) & I (100) & 0 & & & & I (100) & & & & I (100) \\
\hline $\begin{array}{l}\text { S. } \\
\text { liquefasciens }\end{array}$ & I (1.09) & $I(100)$ & 0 & & & I $(100)$ & & & & & I (100) \\
\hline $\begin{array}{l}\text { Salmonella } \\
\text { sp. }\end{array}$ & I (1.09) & $I(100)$ & 0 & & & & & I $(100)$ & & $I(100)$ & \\
\hline TOTAL & $91(100)$ & $53(58.24)$ & $\begin{array}{l}38 \\
(4.75)\end{array}$ & $22(24.17)$ & $19(20.87)$ & $15(16.48)$ & 15 (16.48) & $22(24.17)$ & 38 (4I.75) & $22(24.17)$ & 31 (34.06) \\
\hline
\end{tabular}


Table 2 Distribution of meningitis-causing pneumococcal serotypes by age group

\begin{tabular}{|c|c|c|c|c|c|c|c|c|c|c|c|c|c|c|c|c|c|c|c|c|c|}
\hline $\begin{array}{l}\text { Age } \\
\text { group }\end{array}$ & I & 3 & 5 & 6A/6B & 7F/7A & 8 & II & $12 F$ & 14 & I5B & I7F & 18 & 19 & $23 \mathrm{~F}$ & 24 & 25 & 33 & 35B & NT & Negative & \\
\hline $0-1.5$ & & & 2 & I & 1 & I & & & & & & & & & & & & & 1 & 3 & \\
\hline $1.6-5$ & & 1 & I & 4 & 1 & & 1 & & & & 1 & 2 & 1 & & & & 1 & & 1 & 1 & \\
\hline II-Jun & & 1 & & 1 & & & & I & I & & 1 & & 1 & & & & & 1 & & 1 & \\
\hline 23-Dec & 1 & & & 3 & & & & 1 & & I & & & & & 1 & 1 & & & 1 & & \\
\hline 24-59 & 1 & & & 1 & 1 & & & I & & 2 & & I & & 1 & & & & & & 2 & \\
\hline TOTAL & 2 & 2 & 3 & 10 & 3 & I & 1 & 3 & I & 3 & 2 & 3 & 2 & I & I & I & I & I & 3 & 7 & 51 \\
\hline
\end{tabular}

Table 3 Minimum inhibitory concentrations of tested antibiotics for isolated S. pneumoniae

\begin{tabular}{|c|c|c|c|c|c|c|}
\hline Antibiotics & $\begin{array}{l}\text { CLSI breakpoint } \\
(\mu g / m L)\end{array}$ & $\begin{array}{l}\text { Susceptible } \\
\text { n (\%) }\end{array}$ & Intermediate n (\%) & Resistant n (\%) & MIC $50(\mu \mathrm{g} / \mathrm{ml})$ & MIC90 ( $\mu \mathrm{g} / \mathrm{ml})$ \\
\hline Penicillin G & $R \geq 0.128$ & $20(57.14)$ & & $15(42.85)$ & 0.06 & 0.5 \\
\hline Ceftriaxone & $I=I R \geq 2$ & $31(88.57)$ & I (2.85) & $3(8.57)$ & 0.064 & I \\
\hline Chloramphenicol & $\mathrm{R} \geq 8$ & $26(74.28)$ & & $9(25.7 I)$ & 3 & 24 \\
\hline
\end{tabular}

MIC50, minimum inhibitory concentration required to inhibit the growth of $50 \%$ of isolates; MIC 90 , minimum inhibitory concentration required to inhibit the growth of $90 \%$ of isolates; $R$, resistance breakpoint; I, intermediate breakpoint.

Table 4 Disk diffusion of tested antibiotics for isolated S. pneumoniae

\begin{tabular}{llll}
\hline Antibiotics & Susceptible $\mathbf{n}(\%)$ & Intermediate $\mathbf{n}(\%)$ & Resistant $\mathbf{n}(\%)$ \\
\hline Oxacillin & $17(48.58)$ & 0 & $18(51.42)$ \\
Ampicillin & $32(91.43)$ & 0 & $3(08.57)$ \\
Cephalotin & $29(82.86)$ & 0 & $6(17.14)$ \\
Vancomycin & $35(100)$ & 0 & 0 \\
Érythromycin & $27(77.15)$ & 0 & $8(22.85)$ \\
Clindamycin & $31(88.58)$ & 0 & $4(11.42)$ \\
Ciprofloxacin & $30(85.72)$ & 0 & $5(14.28)$ \\
Levofloxacin & $33(94.28)$ & 0 & $2(05.71)$ \\
Norfloxacin & $30(85.72)$ & 0 & $5(14.28)$ \\
Tetracycline & $13(37.15)$ & $1(02.85)$ & $21(60)$ \\
Doxycyclin & $19(54.3)$ & $1(02.85)$ & $15(42.85)$ \\
Rifampicin & $28(79.16)$ & $2(4.66)$ & $5(16.66)$ \\
Trimethoprim-sulfamethoxazole & $5(14.28)$ & 0 & $30(85.72)$ \\
\hline
\end{tabular}

\section{Discussion}

The mortality rate observed in our study was $16.48 \%$. This was lower than the $27.4 \%$ reported by Bonin ${ }^{21}$ in Cameroon in 1985. It was close to the $21.8 \%$ found by Gervaix ${ }^{7}$ in 2012 and the $18.7 \%$ found by Nguefack. ${ }^{8}$ The most vulnerable age group was those less than one year old, which represented $66.6 \%$ of deaths. This vulnerability within this age group was also found by other authors $(2 ; 12)$. Some $20(21.97 \%)$ children aged less than six weeks (one month and a half) were found to be vulnerable. Because of their age range, they were not eligible for the antipneumococcal vaccination. They may represent a reservoir for pneumococcus. Mezghani in Tunis, over a period of eight years, found $10.7 \%$ cases of meningitis due to $S$. pneumoniae in children less than one month old. ${ }^{22}$ Swann in Malawi found the bacterium in $36.7 \%$ of children less than two months of age. ${ }^{23}$ These results underline the importance of pneumococcal immunization. It is hoped that herd immunity will protect the unimmunized population of young infants less than one-and-a-half months of age. ${ }^{23}$ Both non-PCV13 Serotypes $12 \mathrm{~F}$ and $35 \mathrm{~B}$ meningitis were found from the study. Serotype $12 \mathrm{~F}$ in particular is emerging as an important cause of invasive pneumococcal disease with the propensity to cause outbreaks. ${ }^{10}$

The global resistance profile showed resistance to penicillin $\mathrm{G}$, erythromycin, tetracyclin and trimethoprim-sulfamethoxazole, with PNSSP at $42.85 \%$. These are the antibiotics commonly used in auto-medication as they can be obtained without prescription at non-conventional pharmacies. The extensive use, misuse and abuse of antibiotics have been identified as persistent promoting factors of drug resistance in developing countries, where access to drugs is poorly controlled and the level of self-medication remains high. ${ }^{24}$ 
Susceptibility to penicillin was found to be at $57.15 \%$. The isolates were $91.45 \%$ sensitive to ceftriaxone and $77.15 \%$ sensitive to erythromycin. In a systemic review in Asia, Mamishi found that children younger than five years old were more frequently infected with penicillin-resistant strains. ${ }^{25}$ Iliyasu in Maiduguri (north-east Nigeria) cited Akpede who, in 1994, reported a penicillin nonsusceptible rate of $67 \%$ among pediatric pneumococcal isolates. ${ }^{26}$ In South Africa, from 2003 to 2008, a decrease in sensitivity to penicillin and to ceftriaxone was observed (43 and $8 \%$ ) respectively. ${ }^{27}$ In Malawi, the isolates were all sensitive to ceftriaxone but a recent study suggested that $S$. pneumoniae became less sensitive to this antibiotic. Within the same period in that same site, the sensitivity to erythromycin decreased from $75 \%$ in 2001 to $35 \%$ in $2011 .{ }^{28}$ In Mozambique, $88.2 \%$ of strains were resistant to penicillin while all serotypes were susceptible (100\%) to ceftriaxone. Susceptibility rates for erythromycin, chloramphenicol and tetracycline were also found to be $76.5 \%, 64.7 \%$, and $35.3 \%$, respectively. ${ }^{29}$

In this study, resistance to most other antibiotics was more frequent in the PNSSP isolates than in the susceptible isolates. Pneumococcal antibiotic resistance is even more worrisome in developing countries because PNSSP strains are often multiresistant and because alternative antibiotics (e.g., third-generation cephalosporins) are expensive. Little data exist on empirical treatment guidelines in low-income countries and on the fact that there are high levels of resistance to many commonly used antibiotics. ${ }^{30}$ However, empirical antibiotic usage data revealed empirical prescriptions of trimethoprim-sulfamethoxazole, penicillin and ceftriaxone to which resistance was observed among our isolates, just as in four health facilities in South West Nigeria. ${ }^{24}$ This finding also confirmed the role of antibiotic usage in the emergence and spread of drug-resistant pathogens in endemic populations. Cameroon, like Nigeria, is one of the populations in which antibiotic usage without prescription or with empirical prescription is high. ${ }^{31}$

The current study had many strengths: investigating pneumococcal resistance and mortality, the potentially stable findings, the relatively large and clearly defined study population, the long study duration, the consistency of case definition, and the diagnostic techniques in MCCCBF. Nevertheless, some limitations are acknowledged: all strains could not be tested because of the extreme fragility of pneumococcus that autolyses. Only patients received at the MCC-CBF were included, which does not reflect the general population of Yaoundé. With the introduction and continuous use of antipneumococcal vaccines, notably the 3 free doses of the conjugated 13-valent vaccine in July 2011 in the EPI in Cameroon at 6, 10, and 14 weeks of age, ${ }^{9}$ it is hoped that the proportion of strains resistant to antibiotics will not flourish but decrease. It is important to monitor the resistance of S. pneumoniae to ensure that the recommendations for the presumptive treatment of meningitis and the other Invasive Pneumococcal Diseases (IPD) are taken into account.

\section{Conclusion}

Among children aged less than five years, those less than one year old suffered most from the risk of pneumococcal meningitis, especially those less than six weeks old, who are not eligible for vaccination. The predominance of $S$. pneumoniae was established. The frequency of resistance to penicillin $\mathrm{G}$ was high, while it was moderate for ceftriaxone. Therefore, our findings are usefull for health education and promotion for antibiotic use. We warn against the empirical prescription of antibiotics (particularly drugs like penicillin, trimethoprim-sulfamethoxazole, tetracyclin and chloramphenicol) against $S$. pneumoniae infection in our setting. In a situation where empirical therapy is inevitable, the use of third-generation cephalosporins plus vancomycin is recommended.

\section{Acknowledgments}

We acknowledge the Ministry of Health, Cameroon, the MCC$\mathrm{CBF}$ and the whole team set up for the monitoring of pediatric bacterial meningitis. This includes the WHO, the CDC, the EPI, the DLMEP, the $\mathrm{CPC}$ and the MRC which allowed us to analyze the surveillance data. We especially thank the MCC-CBF that placed its staff, all the necessary reagents, media, antibiotic discs and other materials at our disposal. We also thank Pr. von Gottberg (CRDM-NICD, South Africa), Dr. Mignon du Plessis (CRDM-NICD, South Africa) and Dr. Jean Paul Chedjou (BTC-UYI, Cameroon) for their kind advice.

We acknowledge Pr. Barbara Atogho Tiedeu (French/English Scientific Translator, Interpreter and Reviewer) of Cabinet Competence TI, for reviewing our work. Email: mma_tiedeu@ yahoo.com. Address: 1st Floor Editions CLE Building, 274 Avenue Marechal Foch, P. O. Box 4080, Yaounde, Cameroon.

\section{Conflicts of interest}

Authors have no potential conflicts of interest that could have influenced the present study.

\section{References}

1. Donkor ES. Molecular typing of the pneumococcus and its application in epidemiology in sub-Saharan Africa. Front Cell Infect Microbiol. 2013;3:12.

2. Global health statistics 2012. WHO; 2012.

3. Gessner BD, Mueller JE, Yaro S. African meningitis belt pneumococcal disease epidemiology indicates a need for an effective serotype 1 containing vaccine, including for older children and adults. BMC Infect Dis. 2010;10:22.

4. Weekly epidemiological record. WHO; 2016.

5. Koulla-Shiro S, Kuaban C, Kouda Zeh A, et al. Etiology and outcome of adult bacterial meningitis in Yaounde, Cameroon. Int J Infect Dis. 1997;2(1):9-11.

6. Fonkoua MC, Sorlin P, Musi J, et al. Meningitis of bacterial etiology in Yaoundé (Cameroon) in 1999-2000. Bull Soc Pathol Exot. 2001;4(94):300-303.

7. Gervaix A, Taguebue J, Bescher BN, et al. Bacterial meningitis and pneumococcal serotype distribution in children in Cameroon. Pediatr Infect Dis J. 2012;31(10):1084-1087.

8. Nguefack SCA, Enoh J, Djouberou EH, et al. Etiologies and outcome of children with purulent meningitis at the Yaounde Gyneco-Obstetric and Pediatric Hospital (Cameroon). Open J Ped. 2014;4:269-275.

9. Comprehensive Multiannual Plan 2011-2015 of the Expanded Program on Immunization. MINSANTE; 2011. 110 p.

10. Kwambana ABA, Sarkodie B, Afreh OK, et al. An outbreak of pneumococcal meningitis among older children ( $\geq 5$ years) and adults after the implementation of an infant vaccination programme with the 13-valent pneumococcal conjugate vaccine in Ghana. BMC Infect Dis. 2016;16(1):575.

11. Adiogo D, Ze VN, Beyala F et al. Importance of bacterial resistance in Streptococcus pneumoniae and Streptococcus pyogenes in the center region in Cameroon. Afri J Path Microbiol. 2013;2:1-3. 
12. Shaban L, Siam R. Prevalence and antimicrobial resistance pattern of bacterial meningitis in Egypt. Ann Clin Microbiol Antimicrob. 2009;8:26.

13. Atale A. The decline of antibiotic resistance in dijon nurseries: the combined effect of the correct use of these drugs and the respect of the vaccination calendar. Episante; 2007.

14. Johnson HL, Levine OS, Stoszek SK, et al. Systematic evaluation of serotypes causing invasive pneumococcal disease among children under five: The Pneumococcal Global Serotype Project. PLoS Med. 2010;7(10):e1000348.

15. WHO Coordinated Invasive Bacterial Vaccine Preventable Diseases (IBVPD) Surveillance Network; 2012.

16. Laboratory methods for the diagnosis of meningitis caused by Neisseria meningitis, Streptococcus pneumoniae and Haemophilus influenzae. WHO manual; 2011. 21-31, 76-86 p.

17. Denis F, Martin C, Bingen E, et al. Medical Bacteriology: Usual Techniques. $2^{\text {nd }}$ ed. Elsevier Masson SAS; 2011. 615 p.

18. Performance standards for antimicrobial disk susceptibility tests; Approved standard. Eleventh edition. Wayne, PA: Clinical and Laboratory Standards Institute; 2012.

19. Etest ${ }^{\circledR}$ Antimicrobial Susceptibility Testing. BioMérieux: South Africa; 2012 .

20. Performance standards for antimicrobial susceptibility testing. 26th ed. CLSI supplement M100S. Wayne, PA: Clinical and Laboratory Standards Institute; $2016.93-98$ p.

21. Kago I, Tetanye Ekoe, Tchokoteu PF, et al. Purulent meningitis in Yaoundé: epidemiological and prognostic aspects. Med Infect Dis. 1990;20(10):507-511.

22. Mezghani SM, Kassis M, Mahjoubi Rhimi F, et al. Bacteriology of community meningitis in the Sfax region, Tunisia (1993-2001). Medicine and Infectious Diseases. 2006;36(2):105-110.
23. Swann O, Everett DB, Furyk JS, et al. Bacterial Meningitis in Malawian Infants $<2$ Months of Age: etiology and susceptibility to World Health Organization first-line antibiotics. Pediatr Infect Dis $J$ 2014;33(6):560-565.

24. Bamidele AI, Olubukola A, Afolabi O, et al. A retrospective study of clinical Streptococcus pneumoniae isolates from four health facilities in South-West Nigeria. Int J Med Med Sci. 2012;4(8):160-170.

25. Haghi Ashtiani MT, Sadeghian M, Nikmanesh B, et al. Antimicrobial susceptibility trends among Streptococcus pneumoniae over an 11year period in an Iranian referral children's Hospital. Iran J Microbiol. 2014;6(6):382-386.

26. Lliyasu G, Habib AG, Mohammad AB. Antimicrobial susceptibility pattern of invasive pneumococcal isolates in north west Nigeria. $J$ Glob Infect Dis. 2015;7(2):70-74.

27. Von Mollendorf CCC, de Gouveia L, Quan V et al. Factors associated with ceftriaxone non susceptibility of Streptococcus pneumoniae: Analysis of South African national surveillance data, 2003 to 2010 Antimicrob Agents Chemother. 2014;58(6):3293-3305.

28. Kamng'ona AW, Bar-Zeev N, Gould KA, et al. High multiple carriage and emergence of Streptococcus pneumoniae vaccine serotype variants in Malawian children. BMC Infect Dis. 2015;15:234.

29. Nhantumbo A, Caierão J, Munguambe AM, et al. Serotype distribution and antimicrobial resistance of Streptococcus pneumoniae in children with acute bacterial meningitis in Mozambique: implications for a national immunization strategy. BMC Microbiol. 2016;16(1):134.

30. Ashley E, White NJ, Turner P, et al. Antimicrobial susceptibility of bacterial isolates from community acquired infections in Sub-Saharan Africa and Asian low- and middle-income countries. Trop Med Int Health. 2011;16(9):1167-1179.

31. Obaro SLL, Essen U, Ibrahim K, et al. Community acquired bacteremia in young children from central Nigeria-A pilot study. BMC Infect Dis. $2011 ; 11: 137$ 